\title{
Low Levels of IgG Recognizing the $\alpha$-1-Antitrypsin Peptide and Its Association with Taiwanese Women with Primary Sjögren's Syndrome
}

\author{
Yu-Sheng Chang ${ }^{1,2}$, Chih-Hong Pan ${ }^{3,4}$, Che-Chang Chang 5 (D), Kai-Leun Tsai ${ }^{1}$, \\ Han-Wen Chou ${ }^{6}$, Jin-Hua Chen ${ }^{7,8}$, Sheng-Hong Lin ${ }^{1}$, Yi-Ying Lu ${ }^{6}$, Chih-Chun Tai ${ }^{9}$, \\ Yi-Fang Lin ${ }^{9}$ and Ching-Yu Lin ${ }^{6,10,11, * \text { (D) }}$ \\ 1 Division of Allergy, Immunology, and Rheumatology, Department of Internal Medicine, \\ Shuang Ho Hospital, Taipei Medical University, New Taipei City 23561, Taiwan; \\ risea65@gmail.com (Y.-S.C.); kelen109@hotmail.com (K.-L.T.); koalalin@gmail.com (S.-H.L.) \\ 2 Department of Internal Medicine, School of Medicine, College of Medicine, Taipei Medical University, \\ Taipei 11031, Taiwan \\ 3 Institute of Labor, Occupational Safety and Health, Ministry of Labor, New Taipei City 23561, Taiwan; \\ chpan@mail.ilosh.gov.tw \\ 4 School of Public Health, National Defense Medical Center, Taipei 11031, Taiwan \\ 5 Graduate Institute of Translational Medicine, College of Medical Science and Technology, \\ Taipei Medical University, Taipei 11031, Taiwan; ccchang168@tmu.edu.tw \\ 6 School of Medical Laboratory Science and Biotechnology, College of Medical Science and Technology, \\ Taipei Medical University, Taipei 11031, Taiwan; jessica761022@yahoo.com.tw (H.-W.C.); \\ m609104001@tmu.edu.tw (Y.-Y.L.) \\ 7 Graduate Institute of Data Science, College of Management, Taipei Medical University, Taipei 11031, Taiwan; \\ jh_chen@tmu.edu.tw \\ 8 Research Center of Biostatistics, College of Management, Taipei Medical University, Taipei 11031, Taiwan \\ 9 Department of Laboratory Medicine, Taipei Medical University-Shuang-Ho Hospital, Taipei Medical University, \\ New Taipei City 23561, Taiwan; 08046@s.tmu.edu.tw (C.-C.T.); 08310@s.tmu.edu.tw (Y.-F.L.) \\ 10 Department of Biotechnology and Animal Science, National Ilan University, Ilan 26047, Taiwan \\ 11 Ph.D. Program in Medical Biotechnology, College of Medical Science and Technology, \\ Taipei Medical University, Taipei 11031, Taiwan \\ * Correspondence: cylin@tmu.edu.tw; Tel.: +886-2-2736-1661 (ext. 3326); Fax: +886-2-2732-4510
}

Received: 28 October 2017; Accepted: 12 December 2017; Published: 18 December 2017

\begin{abstract}
The aim of this study was to examine oxidative stress and low level of $\alpha$-1-antitrypsin (A1AT) in primary Sjögren's syndrome (pSS), and evaluate the associated autoreactivity against unmodified and their 4-hydroxy-2-nonenal (HNE)-modified peptides with pSS. Two differentially expressed proteins, $\alpha$-1-acid glycoprotein 1 (A1AG1) and A1AT, exhibited 2-fold differences, and their HNE modifications were identified by depleted-albumin and immunoglobulin $\mathrm{G}(\mathrm{IgG})$ serum protein, in-solution digestion, in-gel digestion, and nano-liquid chromatography-tandem mass spectrometry (nano-LC-MS/MS) from pSS patients and age-matched healthy controls (HCs). Furthermore, levels of proteins, confirmation of HNE modifications, HNE-protein adducts and autoreactivity against unmodified and their HNE-modified peptides were further validated. Levels of the HNE-protein adduct and A1AG1 were significantly higher in pSS patients than HCs, but levels of A1AT were significantly lower in pSS patients compared to HCs. Only the HNE modification of A1AT was confirmed. Our study suggests that elevated HNE-protein adduct, oxidative stress, level (odds ratio (OR) 4.877, $p=0.003$ ), lowered A1AT level (OR 3.910, $p=0.010$ ) and a decreased level of anti-A1AT ${ }^{50-63}$ IgG (OR 3.360, $p=0.010)$ showed an increased risk in pSS patients compared to HCs, respectively.
\end{abstract}

Keywords: primary Sjögren's syndrome; $\alpha$-1-antitrypsin; inhibitor; 4-hydroxy-2-nonenal; autoantibody isotypes; serum 


\section{Introduction}

Primary Sjögren's syndrome (pSS) is a chronic inflammatory autoimmune disease characterized by dysfunction of the exocrine glands leading to dryness of the mouth and eyes [1]. Patients with a pSS feature the presence of autoantibodies mainly against the ribonucleoprotein complex SS-related antigen A (SSA, Ro) and SS-related antigen B (SSB, La) [1]. In 2000-2008, the prevalence of pSS was 16.0 (females 28.8, males 3.7; female:male ratio 7.9) per 100,000 persons; the incidence rate of pSS was 10.6 (females 18.5 , males 2.9 ; female:male ratio 6.3 ) per 100,000 person-years; and the mortality from pSS was 1304.7 (females 987.4, males 3444.2; age-adjusted female:male ratio 0.4) per 100,000 person-years in Taiwan [2]. The etiology and pathogenesis of Sjögren's syndrome are not clearly understood [3]. Jonsson and Brun proposed etiopathogenic events prior to a diagnosis of SS including a genetic predisposition, environmental triggers, autoantibodies, pathological injury, clinical disease, and clinical presentation [4].

Norheim et al. reported that patients with pSS have high levels of oxidative stress compared to healthy controls (HCs) [5]. Wakamatsu et al. found an increase in 4-hydroxy-2-nonenal (HNE)-protein adducts, marker of oxidative stress, in the conjunctiva of SS patients that may play a role in the pathogenesis of dry-eye disease [6,7]. HNE is one of the lipid peroxidation products that has an alkene bond and an aldehyde group which react with amino acid residues that form HNE-protein adducts via types of Michael addition and Schiff-base adducts, respectively [8]. Amino acid residues that can react with HNE include cysteine $(C)$, histidine $(H)$, lysine $(K)$, arginine $(R)$, glutamine $(Q)$, alanine $(A)$, and leucine (L) [8-10]. The HNE-protein adduct is an autoantigen and can elicit specific autoantibody formation [11,12].

Breit et al. indicated that several immune-mediated diseases were associated with an $\alpha$-1-antitrypsin (A1AT) deficiency including rheumatoid arthritis (RA), anterior uveitis, systemic lupus erythematosus (SLE), and asthma in which A1AT may play roles as an anti-inflammatory and immune regulator [13]. A1AT is a serine protease inhibitor [14]. Furthermore, two cases were reported with an A1AT deficiency in patients with pSS in which the A1AT level of plasma decreased by 1.28-2.10-fold [15,16].

In the present study, our aim was to investigate whether a low level of serum A1AT occurs in Taiwanese women with pSS and then identify the HNE modification on A1AT using depleted-albumin and immunoglobulin $\mathrm{G}$ (IgG) serum, in-solution digestion, one-dimensional sodium dodecylsulfate polyacrylamide gel electrophoresis (1D SDS-PAGE), in-gel digestion, and label-free nano-liquid chromatography tandem mass spectrometry (nano-LC-MS/MS) from pSS patients vs. HCs. Furthermore, we also assessed associations of autoantibody isotypes against $\mathrm{A}^{\mathrm{A}} \mathrm{AT}^{50-63}$ and their HNE-modified peptides with pSS patients compared to HCs.

\section{Results}

\subsection{Identification and Validation of Differentially Expressed Serum Proteins by In-Solution Digestion and LC-MS/MS}

Enrichment of depleted-albumin and IgG serum protein samples from a single pair of each of nine pooled serum samples (patients with pSS vs. HCs) was analyzed in triplicate by in-solution digestion coupled to nano-LC-MS/MS (Table 1). In total, 255 proteins were detected, of which 28 differentially expressed proteins significantly varied, as shown in Table 1 and Table S1. There were seven upregulated proteins and 21 downregulated proteins; relative to the HC serum pools, two of the identified proteins, $\alpha$-1-acid glycoprotein 1 (A1AG1) and A1AT, differed by a 2-fold increase or decrease in patients with pSS serum pools, and 26 proteins differed by a 1.7-1.9-fold increase or decrease (Table 1). 
Table 1. Differentially expressed serum proteins identified by in-solution digestion and LC-MS/MS analysis in patients with primary Sjögren's syndrome (pSS) and healthy controls (HCs).

\begin{tabular}{|c|c|c|c|c|c|c|}
\hline \multirow{2}{*}{ Protein ID } & \multirow{2}{*}{ Symbol } & \multirow{2}{*}{ Protein Name } & \multicolumn{2}{|c|}{ Normalized Average Spectral Counts (Mean \pm RSD \%) } & \multirow{2}{*}{$-10 \lg p$} & \multirow{2}{*}{ Fold Change $^{a}$} \\
\hline & & & Normal & pSS & & \\
\hline P02763 & A1AG1 & $\alpha-1$-acid glycoprotein 1 & $1,528,333 \pm 1.76$ & $3,740,000 \pm 6.64$ & 23.5 & $\uparrow 2.4$ \\
\hline P00738 & HPT & Haptoglobin & $730,666,667 \pm 11.29$ & $1,223,333,333 \pm 40.04$ & 44.0 & $\uparrow 1.7$ \\
\hline P69905 & HBA & Hemoglobin subunit $\alpha$ & $56,900,000 \pm 32.80$ & $87,200,000 \pm 9.86$ & 32.4 & $\uparrow 1.5$ \\
\hline P00739 & HPTR & Haptoglobin-related protein & $10,410,000 \pm 3.53$ & $15,366,667 \pm 4.97$ & 30.4 & $\uparrow 1.5$ \\
\hline P01781 & HV320 & Ig heavy chain V-III region GAL & $3,339,000 \pm 1.48$ & $4,910,000 \pm 15.60$ & 22.1 & $\uparrow 1.5$ \\
\hline P68871 & HBB & Hemoglobin subunit beta & $47,033,333 \pm 19.59$ & $63,500,000 \pm 17.09$ & 26.5 & $\uparrow 1.4$ \\
\hline P01860 & IGHG3 & Ig gamma- 3 chain $C$ region & $6,816,667 \pm 10.72$ & $8,310,000 \pm 5.22$ & 23.6 & $\uparrow 1.2$ \\
\hline O75636 & FCN3 & Ficolin-3 & $8,266,667 \pm 3.99$ & $8,640,000 \pm 3.09$ & 27.2 & $\downarrow 1.0$ \\
\hline P01857 & IGHG1 & Ig gamma- 1 chain $C$ region & $161,000,000 \pm 10.64$ & $163,000,000 \pm 7.53$ & 21.6 & $\downarrow 1.0$ \\
\hline P01591 & IGJ & Immunoglobulin J chain & $18,366,667 \pm 5.63$ & $18,300,000 \pm 12.91$ & 21.1 & $\downarrow 1.0$ \\
\hline P02749 & $\mathrm{APOH}$ & Beta-2-glycoprotein 1 & $15,363,333 \pm 3.01$ & $15,266,667 \pm 3.27$ & 25.6 & $\downarrow 1.0$ \\
\hline P02745 & C1QA & Complement $\mathrm{C} 1 \mathrm{q}$ subcomponent subunit $\mathrm{A}$ & $5,913,333 \pm 3.85$ & $5,410,000 \pm 7.56$ & 21.6 & $\downarrow 1.1$ \\
\hline P20742 & PZP & Pregnancy zone protein & $3,450,000 \pm 7.97$ & $3,130,000 \pm 7.19$ & 25.9 & $\downarrow 1.1$ \\
\hline P06681 & $\mathrm{CO} 2$ & Complement $\mathrm{C} 2$ & $2,213,333 \pm 4.01$ & $1,906,667 \pm 11.73$ & 20.6 & $\downarrow 1.2$ \\
\hline P13671 & $\mathrm{CO} 6$ & Complement component $\mathrm{C} 6$ & $4,256,667 \pm 6.68$ & $3,483,333 \pm 9.82$ & 34.0 & $\downarrow 1.2$ \\
\hline P04003 & C4BPA & C4b-binding protein $\alpha$ chain & $38,466,667 \pm 14.64$ & $31,133,333 \pm 7.59$ & 24.3 & $\downarrow 1.2$ \\
\hline P04196 & HRG & Histidine-rich glycoprotein & $30,800,000 \pm 5.97$ & $24,233,333 \pm 18.20$ & 24.9 & $\downarrow 1.3$ \\
\hline P05090 & APOD & Apolipoprotein D & $6,350,000 \pm 5.43$ & $4,936,667 \pm 10.03$ & 26.4 & $\downarrow 1.3$ \\
\hline P01024 & $\mathrm{CO} 3$ & Complement C3 & $175,666,667 \pm 14.62$ & $135,000,000 \pm 9.04$ & 36.3 & $\downarrow 1.3$ \\
\hline P08697 & $\mathrm{A} 2 \mathrm{AP}$ & $\alpha$-2-antiplasmin & $2,643,333 \pm 9.92$ & $1,880,000 \pm 10.03$ & 23.8 & $\downarrow 1.4$ \\
\hline P10909 & CLUS & Clusterin & $20,466,667 \pm 12.03$ & $14,500,000 \pm 11.06$ & 31.1 & $\downarrow 1.4$ \\
\hline P00734 & THRB & Prothrombin & $27,733,333 \pm 16.53$ & $19,566,667 \pm 16.23$ & 27.0 & $\downarrow 1.4$ \\
\hline P09871 & C1S & Complement $\mathrm{C} 1$ s subcomponent & $5,006,667 \pm 6.47$ & $3,423,333 \pm 3.91$ & 36.7 & $\downarrow 1.5$ \\
\hline P00736 & $\mathrm{C} 1 \mathrm{R}$ & Complement $\mathrm{C} 1 \mathrm{r}$ subcomponent & $5,373,333 \pm 9.76$ & $3,450,000 \pm 4.28$ & 37.2 & $\downarrow 1.6$ \\
\hline P02647 & APOA1 & Apolipoprotein A-I & $36,466,667 \pm 33.11$ & $21,866,667 \pm 16.56$ & 44.8 & $\downarrow 1.7$ \\
\hline P02655 & APOC2 & Apolipoprotein C-II & $1,356,667 \pm 53.91$ & $785,000 \pm 4.70$ & 39.7 & $\downarrow 1.7$ \\
\hline P05160 & F13B & Coagulation factor XIII B chain & $431,667 \pm 5.81$ & $228,667 \pm 3.58$ & 21.7 & $\downarrow 1.9$ \\
\hline P01009 & A1AT & $\alpha-1$-antitrypsin & $50,366,667 \pm 8.66$ & $19,766,667 \pm 29.04$ & 72.6 & $\downarrow 2.5$ \\
\hline
\end{tabular}

a Fold change represent increment $(\uparrow)$ and reduction $(\downarrow)$ fold change compared with pSS vs. HC. 
Next, we validated the LC-MS/MS data of A1AG1 and A1AT, and protein levels of A1AG1 $(\sim 48 \mathrm{kDa})$ and A1AT ( 55 kDa) were analyzed by Western blotting (Figure 1). A1AG1 expression levels were significantly higher in pSS samples by 1.53 -fold $(p=0.0001)$ than in HCs, but A1AT levels in pSS samples were significantly lower than those in HCs by 1.84 -fold ( $p=0.0071$, Figure $1 \mathrm{~A}, \mathrm{~B}$, right upper panel). Equal amounts of serum proteins in these experiments were examined (Figure 1A,B, right bottom panel). The area under the ROC curve (AUC) value, sensitivity, and specificity of serum A1AG1 and A1AT in pSS samples vs. HCs were calculated based on these results and plotted on an ROC curve. The Western blot results of A1AG1 showed that the AUC was 0.75, the sensitivity was $85.0 \%$, and the specificity was $62.5 \%$ for pSS detection at an average densitometric cutoff of 19,994.82; the AUC was 0.67 , the sensitivity was $77.5 \%$, and the specificity was $60.0 \%$ for pSS detection by A1AT at an average densitometric cutoff of 104,087.25 (Figure 1C).

A


B


Figure 1. Cont. 
C



Figure 1. Protein levels of A1AG1 and A1AT in serum were examined using anti-A1AG1 (A) and anti-A1AT (B) antibodies through Western blotting. Average blot densitometric values were calculated from duplicate data. Percentages of sodium dodecyl sulfate polyacrylamide gel electrophoresis (SDS-PAGE) gel and loading amounts of serum proteins used in Western blotting were 10\% and $2 \mu \mathrm{g}$ for A1AG1, and $8 \%$ and $2 \mu \mathrm{g}$ for A1AT, respectively. A duplicate gel was stained with Coomassie brilliant blue (CBB) as a loading control (right, bottom panel). The red arrow indicates the A1AG1 or A1AT protein. Receiver operating characteristic (ROC) curves were generated according to blot densitometry of A1AG1 and A1AT. The area under the ROC curve (AUC), sensitivity, and specificity were further estimated (C).

\subsection{Novel HNE Modification Identification of Serum Proteins by In-Gel Digestion and LC-MS/MS}

In addition to serum protein levels, we further identified HNE modifications of A1AG1 and A1AT. The average coverage of amino acid sequences in A1AG1 and A1AT were estimated to be $52 \%$ and $70 \%$, respectively. No HNE modification was identified on A1AG1 (Table S2A). Novel HNE modifications of A1AT were identified by manual examination of the modified spectra using the PeaksPTM module in PEAKS 7 software (version 7.0, Bioinformatics Solutions, Waterloo, ON, Canada). Furthermore, HNE modifications of A1AT were confirmed in the two pooled serum samples (patients with pSS vs. HCs) through IP-Western blotting, which detected signals of approximately $55 \mathrm{kDa}$ (Figure 2). Because low coverage of A1AG1 was identified, we also confirmed HNE modifications of A1AG1 using IP-Western blotting, but no signal was detected (Table S2B).

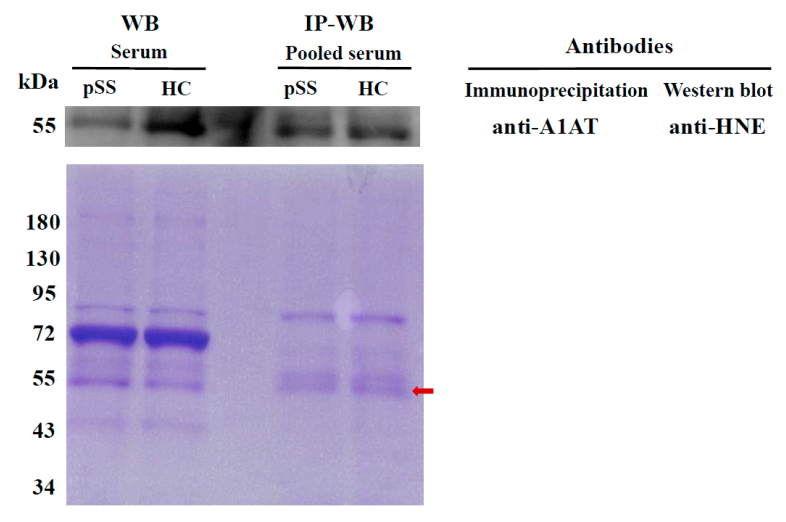

Figure 2. 4-Hydroxy-2-nonenal (HNE) modification of the serum A1AT protein was validated using immunoprecipitation (IP) and Western blotting. A1AT was immunoprecipitated from pooled serum samples (40 patients with primary Sjögren's syndrome (pSS) and 40 healthy controls (HCs)) using anti-A1AT antibodies and then subjected to Western blotting with anti-HNE antibodies (upper panel). Individually selected random serum samples (patient with pSS and HC) were used as controls; these were simultaneously used for Western blotting with anti-HNE antibodies. A duplicate gel was stained with Coomassie brilliant blue as a loading control (bottom panel). The red arrow indicates the A1AT protein. 
MS/MS spectrum data of HNE-modified peptides on A1AT are presented in Figure S1A and Table S3. The peptide ${ }^{50}$-ITPNLAEFAFSLYR-63 was used to identify A1AT as pSS-specific and was found to have an HNE modification at A58. The peptide moiety was identified on the basis of $b$ - and y-series ions. The HNE-modified residue of peptide was confirmed a mass increase of 156.11504 Da through an unmodified b8 ion followed by a modified y6 ion. (Figure S1B, upper panel). The peptide ${ }^{360}$-AVLTIDEK- 367 was used to identify A1AT as HC-specific and was found to have an HNE modification at A360. A mass increase of 138.10446 Da of HNE-modified residues in peptide was identified through an unmodified y7 ion followed by a modified b1 ion. (Figure S1B, bottom panel).

\subsection{Autoreactivity against $A 1 A T^{50-63}$ and $A 1 A T^{50-63}$ HNE Peptides}

Serum samples were assessed with autoantibody isotypes against $\mathrm{A}^{\mathrm{A}} \mathrm{AT}^{50-63}$ and $\mathrm{A} 1 \mathrm{AT} \mathrm{T}^{50-63} \mathrm{HNE}$ peptides by an enzyme-linked immunosorbent assay (ELISA). The analysis of variance (ANOVA) statistical analysis revealed that the difference in anti-A1AT ${ }^{50-63} \operatorname{IgG}$ antibody $(p<0.0001)$ and anti-A1AT ${ }^{50-63}$ HNE IgG antibody $(p<0.0001)$ was significant among patients with pSS, RA and SLE, and HCs (Figure 3). The level of the anti-A1AT ${ }^{50-63} \mathrm{IgG}$ antibody in RA was significantly higher than that of HCs by 1.80-fold ( $p=0.0006)$, that of SLE vs. HC was 2.41-fold higher $(p<0.0001)$, that of RA vs. pSS was 2.52 -fold higher $(p<0.0001)$, that of SLE vs. pSS was 3.38-fold higher $(p<0.0001)$; however, that of SLE vs. RA was not significantly 1.34-fold higher and that of pSS was not significantly lower than that of the HCs by 1.40-fold (Figure 3A, left panel). Anti-A1AT ${ }^{50-63} \operatorname{IgM}$ and anti-A1AT ${ }^{50-63} \mathrm{IgA}$ expression levels did not significantly differ among patients with pSS, RA, SLE, and HCs (Figure 3A, middle panel, right panel).

A



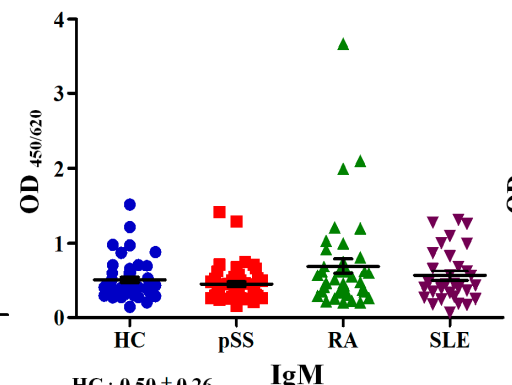

HC: $0.50 \pm 0.26$ $\mathrm{pSS}: 0.44 \pm 0.24$ RA : $0.69 \pm 0.64$ SLE : $0.56 \pm 0.36$ A1AT $^{50-63}$ HNE
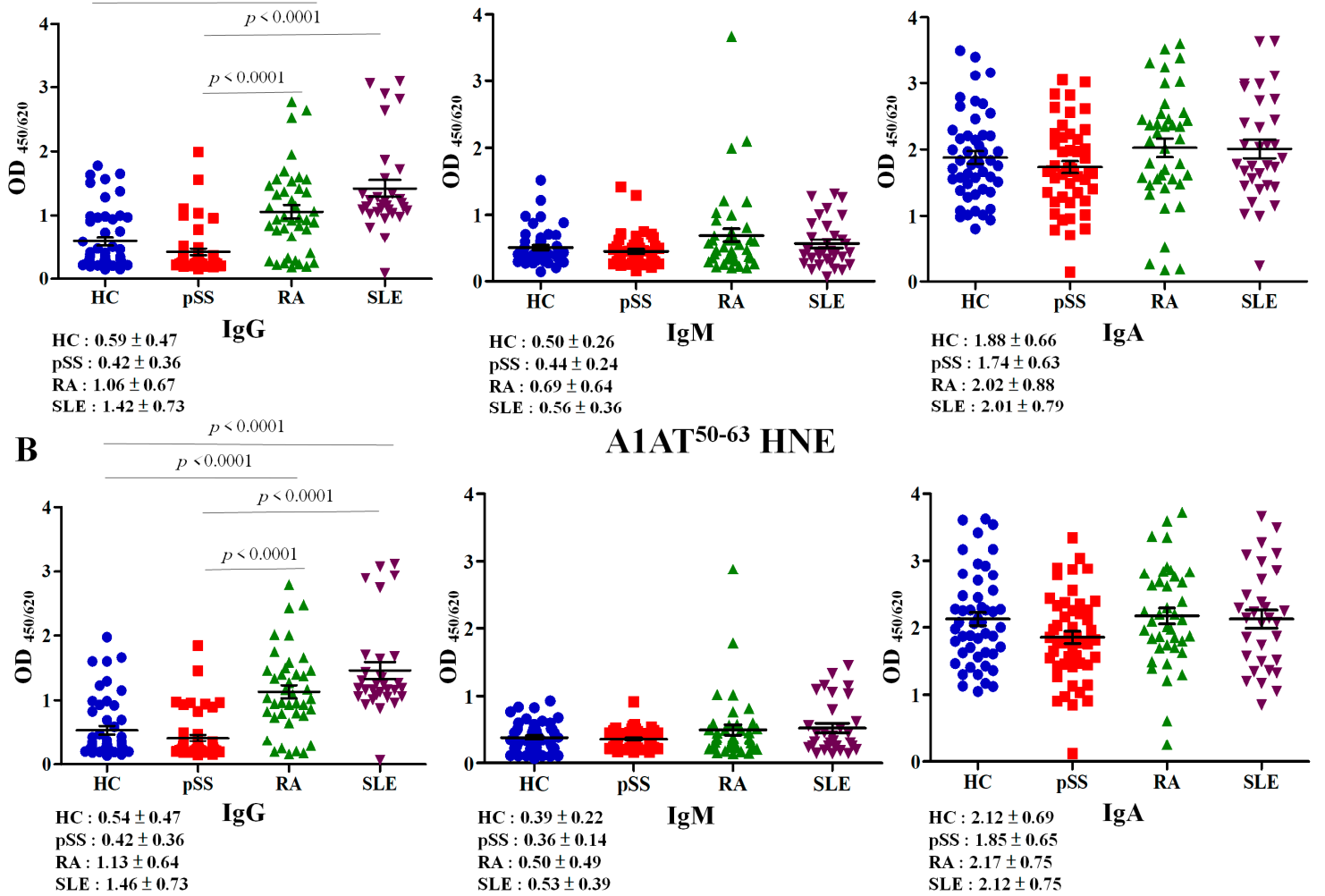

Figure 3. Dot plot of serum concentrations (absorbance units at 450/620 nm) of IgG, IgM, and $\operatorname{IgA}$ autoantibody isotypes recognizing $\mathrm{A}_{1} \mathrm{AT}^{50-63}$ (A) and $\mathrm{A}^{\mathrm{A}} \mathrm{AT}^{50-63}$ 4-hydroxy-2-nonenal (HNE) (B) in healthy controls (HCs), patients with primary Sjögren's syndrome (pSS), rheumatoid arthritis (RA), and systemic lupus erythematosus (SLE) with an ELISA. OD $450 / 620$, optical density at 450/620 nm. 
The level of the anti-A1AT ${ }^{50-63}$ HNE IgG antibody in RA was significantly higher than that of HCs by 2.10 -fold $(p<0.0001)$, that of SLE vs. HC was 2.70 -fold higher $(p<0.0001)$, that of RA vs. pSS was 2.69-fold higher $(p<0.0001)$, that of SLE vs. pSS was 3.48-fold higher $(p<0.0001)$; however, that of SLE vs. RA was not significantly and that of pSS did not significantly differ from that of the HCs (Figure 3B, left panel). Anti-A1AT ${ }^{50-63} \mathrm{HNE}$ IgM and Anti-A1AT ${ }^{50-63} \mathrm{HNE}$ IgA expression levels did not significantly differ among patients with pSS, RA, SLE, and HCs (Figure 3B, middle panel, right panel).

\subsection{Determination of HNE-Protein Adducts}

The level of the HNE-protein adduct can present the oxidative stress status and plays important pathogenic roles in several diseases including cancer, and neurodegenerative, chronic inflammatory, and autoimmune diseases [17]. As shown in Table S4, serum levels of the HNE-protein adduct in pSS patients were significantly higher compared to those of the HCs (1.27-fold, $p=0.0004)$.

\subsection{Association of Elevated HNE-Protein Adduct, Lowed A1AT Level or Decreased Autoreactivity against} $A 1 A T^{50-63}$ and $A 1 A T^{50-63}$ HNE Peptides with pSS Patients

In Table 2, HNE-protein adduct, serum A1AT and anti-A1AT ${ }^{50-63}$ IgG of pSS patients carried a 4.887 -fold risk $(p=0.003$, power $=0.708), 3.910$-fold risk $(p=0.010$, power $=0.726)$ and a 3.360 -fold risk $(p=0.010$, power $=0.802)$ showed a significant difference compared to HCs after adjusting for age, in the logistic regression analyses, respectively. Risks did not significantly differ after the age-adjusted logistic regression, suggesting that they were associated with other low levels of autoantibodies (Table 2).

Table 2. Association among HNE-protein adduct, A1AT, anti-A1AT ${ }^{50-63}$ and their HNE-modified peptides antibodies and pSS patients, in patients with pSS vs. healthy controls.

\begin{tabular}{|c|c|c|c|c|c|c|}
\hline \multirow{2}{*}{ Risk Factor } & \multirow{2}{*}{ Cut-Off } & \multirow{2}{*}{$\mathrm{HC}, n$} & \multirow{2}{*}{ pSS, $n$} & \multicolumn{2}{|c|}{ Age-Adjusted Logistic Regression Model a } & \multirow{2}{*}{ Power } \\
\hline & & & & ORs $(95 \% \mathrm{CI})$ & $p$ Value & \\
\hline \multirow{2}{*}{ HNE-protein adduct } & $\geq 2.884$ & 29 & 43 & 1.0 & & 0.708 \\
\hline & $<2.884$ & 20 & 6 & $4.887(1.744-13.637)$ & 0.003 & \\
\hline \multirow{2}{*}{ Serum A1AT } & $\geq 123,574.37$ & 19 & 7 & 1.0 & & 0.726 \\
\hline & $<123,574.37$ & 21 & 33 & $3.910(1.384-11.035)$ & 0.010 & \\
\hline \multirow{2}{*}{ Anti-A1AT ${ }^{50-63} \mathrm{IgG}$} & $\geq 0.407$ & 21 & 9 & 1.0 & & \\
\hline & $<0.407$ & 28 & 40 & $3.360(1.336-8.451)$ & 0.010 & 0.802 \\
\hline \multirow{2}{*}{ Anti-A1AT ${ }^{50-63} \mathrm{HNE}$ IgG } & $\geq 0.350$ & 22 & 13 & 1.0 & & \\
\hline & $<0.350$ & 27 & 36 & $2.263(0.967-5.299)$ & 0.060 & 0.500 \\
\hline \multirow{2}{*}{ Anti-A1AT ${ }^{50-63} \operatorname{IgA}$} & $\geq 1.796$ & 25 & 19 & 1.0 & & \\
\hline & $<1.796$ & 24 & 30 & $1.650(0.738-3.687)$ & 0.222 & 0.232 \\
\hline \multirow{2}{*}{ Anti-A1AT ${ }^{50-63}$ HNE IgA } & $\geq 1.903$ & 28 & 21 & 1.0 & & \\
\hline & $<1.903$ & 21 & 28 & $1.786(0.801-3.986)$ & 0.157 & 0.293 \\
\hline
\end{tabular}

\section{Discussion}

This is the first study to investigate the association between decreased serum levels of autoantibody isotypes against $\mathrm{A}_{1} \mathrm{AT}^{50-63}$ and $\mathrm{A} 1 \mathrm{AT}^{50-63} \mathrm{HNE}$ peptides and the risk of low A1AT levels in pSS patients. In the present study, two differentially expressed proteins, A1AG1 and A1AT, had 2-fold differences in depleted-albumin and IgG serum protein pools of nine pSS patients vs. nine HCs, identified in triplicate from in-solution digestion coupled to LC-MS/MS (Table 1). However, A1AG1 (1.53-fold increase, AUC = 0.75) and A1AT (1.84-fold decline, AUC $=0.67$ ) showed acceptable diagnostic values for discriminating between pSS patients and HCs according to a Western blot analysis (Figure 1C). In this study, significantly higher serum levels of HNE-protein adducts indicated 
increments in the oxidative stress status of pSS patients (Table S4); these results are consistent with those of previous studies $[5,6]$.

A1AG is an acute-phase protein, and its serum levels are elevated in response to a local inflammatory stimulus in several diseases including depression, cancer, and acquired autoimmune deficiency syndrome [18]. A1AG may have anti-inflammatory and immunomodulatory properties [19]. In the macrophage deactivation process, A1AG1 may act as a signaling molecule in the maintenance of tissue homeostasis and remodeling [20]. Rantapaa-Dahlqvist et al. reported that pSS patients with pericarditis had significantly higher levels of A1AG than did pSS patients without pericarditis [21]; however, no information on A1AG1's involvement in the development of pSS has been reported. In this study, serum protein levels of A1AG1 in pSS were significantly higher than those of HCs by 1.53-fold (Figure 1A). Saroha et al. indicated that altered glycosylation and expression of plasma A1AG may play a role in RA progression [22].

$\mathrm{A} 1 \mathrm{AT}$ is also an acute-phase protein that has anti-inflammatory and tissue-protective properties and is an immune regulator $[13,23]$. Human A1AT protein levels can increase to inhibit elastase and serine-type proteinase during inflammation [14]. Serum protein levels of A1AT in patients with pSS were significantly lower than those of HCs by 1.84-fold (Figure 1B); these results are consistent with previous studies $[15,16]$. In this study, patients with pSS showed a feature of low A1AT level (Figure 1B). Thus, a low serum level of A1AT is a risk factor for the development of pSS (Table 2). However, Elshikha et al. demonstrated that the human A1AT protein has protective effects through inhibition of dendritic cell (DC) activation and function to attenuate autoimmunity in RA mouse models [24]. Ciobanu et al. indicated that significantly lower levels of A1AT in rheumatoid synovial fluid can decrease the anti-protease activity in RA [25]. Stefanescu et al. showed that levels of anti-A1AT antibodies were significantly elevated in RA [26]. In several previous studies, elevated IgA-A1AT complex levels were reported in RA, SLE, mixed connective tissue disease, and ankylosing spondylitis compared to HCs [27-29]. Furthermore, Lacki et al. suggested that a high level of the IgA-A1AT complex may cause worsening of bone erosion in RA [29]. In this study, levels of IgA-A1AT ${ }^{50-63}$ and their HNE-modified peptide complexes did not significantly differ among patients with RA and SLE compared to HCs (Figure 3A,B, right panel). Levels of IgG-A1AT ${ }^{50-63}$ and their HNE-modified peptide complexes were significantly higher among patients with RA and SLE compared to HCs (Figure 3A,B, left panel). However, serum levels of anti-A1AT ${ }^{50-63}$ IgG and anti-A1AT ${ }^{50-63}$ HNE IgG were not significantly lower in pSS patients (Figure 3). Furthermore, we observed that low levels of the anti-A1AT ${ }^{50-63} \mathrm{IgG}$ antibody may be an increased risk against pSS (Table 2). The presence of self-reactive IgG autoantibodies in human serum is thought to represent as pathogenic antibodies in patients with pSS [30]. Furthermore, the HNE-modified epitope belongs to oxidation-specific epitopes (OSEs) [31]. OSEs are present on damaged proteins and induce specific autoantibodies formation [11,12]. Anti-OSE autoantibodies have conveyed protection from autoimmune pathogenesis [30,32,33]. Importantly, oxidative stress remained in patients with pSS (Table S4).

\section{Materials and Methods}

\subsection{Patient Samples}

This study was approved by the institutional review board of the study hospital, and all volunteers provided informed consent before being allowed to participate (No. 201501059, 2015/05/09, TMU-Joint Institutional Review Board). Serum samples from 168 female patients (49 with pSS (55.50 \pm 12.85 years old), 40 with RA ( $54.30 \pm 11.30$ years old), and 30 with SLE (40.60 \pm 11.18 years old)) and 49 age-matched female HCs (55.40 \pm 11.67 years old) were obtained from the Division of Allergy, Immunology and Rheumatology, Department of Internal Medicine and Department of Laboratory Medicine, Shuang-Ho Hospital (New Taipei City, Taiwan). Patients with pSS, RA, or SLE were diagnosed by a rheumatologist and had satisfied appropriate classification criteria. RA patients had received a diagnosis from a rheumatologist and had fulfilled appropriate classification 
criteria-either the 2010 American College of Rheumatology (ACR)/European League Against Rheumatism classification criteria [34] or the 1987 ACR classification criteria [35]. pSS patients were diagnosed according to the American-European Consensus Group (AECG) classification criteria [36]. SLE patients fulfilled the 1997 ACR SLE classification criteria [37]. Differentially expressed serum proteins were identified through in-solution digestion and nano-LC-MS/MS using pooled depleted-albumin and IgG serum protein samples randomly selected from nine RA patients and nine age-matched HCs. Two differentially expressed proteins, A1AG1 and A1AT, exhibited 2-fold differences in patients with pSS compared to HCs, and these were selected to examine protein levels through Western blotting using individual serum samples randomly selected from another 40 pSS patients and 40 age-matched HCs. HNE modifications of A1AT and A1AG1 were identified by in-gel digestion and nano-LC-MS/MS. HNE modifications of proteins were evaluated through IP and Western blotting using the aforementioned 40 pairs of pooled serum samples. Next, autoantibody isotypes against unmodified and their HNE-modified peptides were assessed among 49 pSS, $40 \mathrm{RA}$, and $30 \mathrm{SLE}$ patients, and $49 \mathrm{HCs}$. Serum was stored at $-20^{\circ} \mathrm{C}$ until being analyzed. Clinical and demographic characteristics of pSS, RA, and SLE patients, and HCs are presented in Table S4. However, the age of patients with SLE was significantly lower compared to those of the pSS, RA, and HC cohorts (Table S4).

\subsection{Depleted-Albumin and IgG Serum Proteins, In-Solution Digestion, and Protein Identification by LC-MS/MS}

Protein concentrations of serum were determined using a Coomassie Plus (Bradford) Assay Kit according to the manufacturer's protocol. Albumin and IgG of serum samples were removed using an Albumin and IgG Depletion SpinTrap column according to the protocol of Uen et al. [38]. Three micrograms of depleted-albumin and IgG serum proteins were used to perform in-solution digestion using an In-Solution Tryptic Digestion and Guanidination Kit according to the manufacturer's instructions. Tryptic peptide mixtures were analyzed in triplicate using NanoLC-nanoESi-MS/MS that was performed on a nanoAcquity system (Waters, Milford, MA, USA) connected to an LTQ-Orbitrap $\mathrm{XL}^{\mathrm{TM}}$ hybrid mass spectrometer (Thermo Fisher Scientific, Bremen, Germany) equipped with a nanospray interface (Proxeon, Odense, Denmark). Differentially expressed proteins were quantified using label-free peptide quantification by the Peaks Q module of the PEAKS 7 software (version 7.0, Bioinformatics Solutions, Waterloo, ON, Canada) [39]. Details are provided in "Supplementary Information".

\subsection{Western Blotting}

Serum protein levels of differentially expressed proteins showing 2-fold differences in pSS patients vs. HCs were examined using a Western blot analysis. A1AG1 ( $2 \mu \mathrm{g}$ of protein in $10 \%$ SDS-PAGE) or A1AT ( $2 \mu \mathrm{g}$ of protein in $8 \%$ SDS-PAGE) was evaluated using a mouse anti-A1AG1 monoclonal antibody (sc-69753, Santa Cruz Biotechnology, Dallas, TX, USA) or a mouse anti-A1AT monoclonal antibody (sc-69752, Santa Cruz Biotechnology). Details are provided in "Supplementary Information".

\subsection{1-D SDS-PAGE, In-Gel Digestion, and HNE Identification by LC-MS/MS}

Fifty-microgram protein samples (pooled serum proteins of A1AG1 or A1AT) were run on $10 \%$ SDS-PAGE with in-gel digestion according to a previously described method with minor modifications (Figure S1C) [40]. HNE modifications were identified in triplicate using tryptic peptide mixtures of gel slices by the aforementioned nano-LC-MS/MS (nanoAcquity system and LTQ-Orbitrap XL ${ }^{\text {TM }}$ hybrid mass spectrometer). The PeaksPTM module of the PEAKS 7 software (Bioinformatics Solutions) was used to identify HNE-modified peptide sequences and sites of serum A1AG1 and A1AT. Details are provided in "Supplementary Information".

\subsection{IP}

An IP experiment for A1AG1 or A1AT was performed using a mouse anti-A1AG1 monoclonal antibody (sc-69753, Santa Cruz Biotechnology) or a mouse monoclonal antibody (sc-69752, Santa Cruz 
Biotechnology). HNE modifications of A1AG1 or A1AT were evaluated through a Western blot analysis with a goat polyclonal anti-HNE antibody (MBS536107, MyBioSource, San Diego, CA, USA). Details are provided in "Supplementary Information".

\subsection{Detection of Autoreactivity against $A 1 A T^{50-63}$ and Their HNE-Modified Peptides}

Polypeptides corresponding to the 50-63 amino acid sequence of human A1AT, i.e., ITPNLAEFAFSLYR (named A1AT ${ }^{50-63}$ ) were synthesized (Yao-Hong Biotechnology, New Taipei City, Taiwan) and their HNE-modified A1AT ${ }^{50-63}$ (named A1AT ${ }^{50-63} \mathrm{HNE}$ ) used in an ELISA. In total, 168 serum samples were assessed for the presence of $\operatorname{IgG}, \operatorname{IgM}$, and IgA isotypes of anti-A1AT ${ }^{50-63}$ and anti-A1AT ${ }^{50-63}$ HNE peptide antibodies. The absorbance was measured at $450 \mathrm{~nm}$ with the reference filter set to $620 \mathrm{~nm}$. All samples were treated in duplicate. Details are provided in "Supplementary Information".

\subsection{Detection of Serum HNE-Protein Adducts}

Levels of HNE-protein adducts were quantified using 168 serum samples for the ELISA protocol of Weber et al. [41]. All samples were analyzed in duplicate. Details are provided in "Supplementary Information".

\subsection{Statistical Analyses}

Student's $t$-test was used to determine the significance of differences in blot densitometry, levels of serum proteins, and levels of HNE-protein adducts. A one-way ANOVA was used to test levels of autoantibody isotypes against $\mathrm{A}^{\mathrm{A}} \mathrm{T}^{50-63}$ and $\mathrm{A} 1 \mathrm{AT} \mathrm{T}^{50-63} \mathrm{HNE}$ peptides among patients with $\mathrm{pSS}$, RA and SLE, and HCs. Scheffe's post hoc test were used to compare the mean difference between any two disease groups. Post hoc test by Bonferroni method with 0.0083 adjusted significance level. GraphPad Prism (version 5.0, Graphpad Software, San Diego, CA, USA) was used to assess differences in Student's $t$-tests and a dot plot was drawn. All results are shown as the mean \pm standard deviation (SD) except Spectral count data that is presented as the mean \pm relative SD (RSD). The RSD is a coefficient of variation (CV) and is calculated as a percentage. Multiples of change were defined as (mean of pSS-normalized spectral counts)/(mean of HC-normalized spectral counts). The threshold for up- or downregulated proteins was a 1.0-fold change in expression. Comparisons of pSS vs. HC serum samples were performed. Proteins that had a 2 -fold difference were selected for validation by a Western blot analysis. Univariate and multiple logistic regression models were further used to estimate the adjusted odds ratios (ORs) and their 95\% confidence intervals (CIs) for the pSS risk. Power and ANOVA are estimated using SAS (version 9.3, SAS Institute, Cary, NC, USA). The diagnostic performance of differentially expressed proteins was evaluated using receiver operating characteristic (ROC) curves by MedCalc Statistical Software (version 15.4, MedCalc Software, Ostend, Belgium). The $95 \%$ confidence level of the area under the ROC curve (AUC), sensitivity, and specificity were calculated. The significance level of statistical tests was set to $p$-value, which was less than 0.05 .

\section{Conclusions}

We identified HNE modifications on the human serum A1AT protein in vivo to investigate autoantibody isotypes against $\mathrm{A} 1 \mathrm{AT}^{50-63}$ and $\mathrm{A} 1 \mathrm{AT}^{50-63} \mathrm{HNE}$ peptides associated with pSS patients. Our results showed that low levels of the anti-A1AT ${ }^{50-63} \mathrm{IgG}$ antibody may have an increased risk in pSS patients. However, this possibility needs to be confirmed in larger studies.

Supplementary Materials: The following are available online at www.mdpi.com/1422-0067/18/12/2750/s1.

Acknowledgments: This study was supported by a grant (MOST104-2314-B-038-026) from the Ministry of Science and Technology, City, Taiwan. Proteomics data were analyzed by the Academia Sinica Common Mass Spectrometry Facilities on an LTQ-Orbitrap XL hybrid mass spectrometer located at the Institute of Biological Chemistry (Taipei, Taiwan). The authors thank Tzu-Yun Yu, Research Center of Biostatistics, College of Management of Taipei Medical University, Taiwan, who provided consultant services for power calculations. 
Author Contributions: Ching-Yu Lin, Che-Chang Chang, Yu-Sheng Chang, Kai-Leun Tsai and Sheng-Hong Lin conceived and designed the experiments; Han-Wen Chou, Chih-Chun Tai and Yi-Fang Lin performed the experiments; Jin-Hua Chen, Chih-Hong Pan, Yi-Ying Lu and Han-Wen Chou analyzed the data; Ching-Yu Lin and Che-Chang Chang wrote the paper.

Conflicts of Interest: The authors declare no conflict of interest.

\section{Abbreviations}

pSS
RA
SLE
HNE
1-D SDS-PAGE
nano-LC-MS/MS
HC
Ig
ACR
IP

A1AG1

RSD

$\mathrm{SD}$

ROC

AUC

CBB

RF

ANA

anti-Ro (SSA)

anti-La (SSB)

CRP

ESR

OR

ESSDAI

NSAIDs

DMARDs

DMARDs



\section{References}

1. Jonsson, R.; Vogelsang, P.; Volchenkov, R.; Espinosa, A.; Wahren-Herlenius, M.; Appel, S. The complexity of Sjögren's syndrome : Novel aspects on pathogenesis. Immunol. Lett. 2011, 141, 1-9. [CrossRef] [PubMed]

2. Yu, K.H.; See, L.C.; Kuo, C.F.; Chou, I.J.; Chou, M.J. Prevalence and incidence in patients with autoimmune rheumatic diseases: A nationwide population-based study in Taiwan. Arthritis Care Res. 2013, 65, 244-250. [CrossRef] [PubMed]

3. Maslinska, M.; Przygodzka, M.; Kwiatkowska, B.; Sikorska-Siudek, K. Sjögren's syndrome: Still not fully understood disease. Rheumatol. Int. 2015, 35, 233-241. [CrossRef] [PubMed]

4. Jonsson, R.; Brun, J.G. Sjögren's syndrome. In Els; John Wiley \& Sons, Ltd.: Hoboken, NJ, USA, 2010.

5. Norheim, K.B.; Jonsson, G.; Harboe, E.; Hanasand, M.; Goransson, L.; Omdal, R. Oxidative stress, as measured by protein oxidation, is increased in primary Sjögren's syndrome. Free Radic. Res. 2012, 46, 141-146. [CrossRef] [PubMed]

6. Wakamatsu, T.H.; Dogru, M.; Matsumoto, Y.; Kojima, T.; Kaido, M.; Ibrahim, O.M.; Sato, E.A.; Igarashi, A.; Ichihashi, Y.; Satake, Y.; et al. Evaluation of lipid oxidative stress status in Sjögren's syndrome patients. Investig. Ophthalmol. Vis. Sci. 2013, 54, 201-210. [CrossRef] [PubMed]

7. Uchida, K. Protein-bound 4-hydroxy-2-nonenal as a marker of oxidative stress. J. Clin. Biochem. Nutr. 2005, 36, 1-10. [CrossRef] 
8. Zhao, J.; Chen, J.; Zhu, H.; Xiong, Y.L. Mass spectrometric evidence of malonaldehyde and 4-hydroxynonenal adductions to radical-scavenging soy peptides. J. Agric. Food Chem. 2012, 60, 9727-9736. [CrossRef] [PubMed]

9. Lv, L.; Lin, H.; Li, Z.; Yuan, F.; Gao, Q.; Ma, J. Effect of 4-hydroxy-2-nonenal treatment on the IgE binding capacity and structure of shrimp (Metapenaeus ensis) tropomyosin. Food Chem. 2016, 212, 313-322. [CrossRef] [PubMed]

10. Uchida, K. 4-hydroxy-2-nonenal: A product and mediator of oxidative stress. Prog. Lipid Res. 2003, 42, 318-343. [CrossRef]

11. Eggleton, P.; Nissim, A.; Ryan, B.J.; Whiteman, M.; Winyard, P.G. Detection and isolation of human serum autoantibodies that recognize oxidatively modified autoantigens. Free Radic. Biol. Med. 2013, 57, $79-91$. [CrossRef] [PubMed]

12. Alzolibani, A.A.; Al Robaee, A.A.; Al-Shobaili, H.A.; Rasheed, Z. 4-hydroxy-2-nonenal modified histone-H2A: A possible antigenic stimulus for systemic lupus erythematosus autoantibodies. Cell. Immunol. 2013, 284, 154-162. [CrossRef] [PubMed]

13. Breit, S.N.; Wakefield, D.; Robinson, J.P.; Luckhurst, E.; Clark, P.; Penny, R. The role of $\alpha$ 1-antitrypsin deficiency in the pathogenesis of immune disorders. Clin. Immunol. Immunopathol. 1985, 35, 363-380. [CrossRef]

14. Macen, J.L.; Upton, C.; Nation, N.; McFadden, G. SERP1, a serine proteinase inhibitor encoded by myxoma virus, is a secreted glycoprotein that interferes with inflammation. Virology 1993, 195, 348-363. [CrossRef] [PubMed]

15. Lindstrom, F.D.; Skogh, T.; Lundstrom, I.M. $\alpha 1$ antitrypsin deficiency in a patient with systemic vasculitis and primary Sjögren's syndrome. Ann. Rheum. Dis. 2002, 61, 945-946. [CrossRef] [PubMed]

16. Malfait, R.; Gorus, F.; Sevens, C. Electrophoresis of serum protein to detect $\alpha 1$-antitrypsin deficiency: Five illustrative cases. Clin. Chem. 1985, 31, 1397-1399. [PubMed]

17. Barrera, G.; Pizzimenti, S.; Ciamporcero, E.S.; Daga, M.; Ullio, C.; Arcaro, A.; Cetrangolo, G.P.; Ferretti, C.; Dianzani, C.; Lepore, A.; et al. Role of 4-hydroxynonenal-protein adducts in human diseases. Antioxid. Redox Signal. 2015, 22, 1681-1702. [CrossRef] [PubMed]

18. Fournier, T.; Medjoubi, N.N.; Porquet, D. $\alpha 1$-acid glycoprotein. Biochim. Biophys. Acta 2000, 1482, $157-171$. [CrossRef]

19. Hochepied, T.; Berger, F.G.; Baumann, H.; Libert, C. $\alpha 1$-acid glycoprotein: An acute phase protein with inflammatory and immunomodulating properties. Cytokine Growth Factor Rev. 2003, 14, 25-34. [CrossRef]

20. Gemelli, C.; Martello, A.; Montanari, M.; Zanocco Marani, T.; Salsi, V.; Zappavigna, V.; Parenti, S.; Vignudelli, T.; Selmi, T.; Ferrari, S.; et al. The orosomucoid 1 protein is involved in the vitamin D-mediated macrophage de-activation process. Exp. Cell Res. 2013, 319, 3201-3213. [CrossRef] [PubMed]

21. Rantapaa-Dahlqvist, S.; Backman, C.; Sandgren, H.; Ostberg, Y. Echocardiographic findings in patients with primary Sjogren's syndrome. Clin. Rheumatol. 1993, 12, 214-218. [CrossRef] [PubMed]

22. Saroha, A.; Biswas, S.; Chatterjee, B.P.; Das, H.R. Altered glycosylation and expression of plasma $\alpha 1$-acid glycoprotein and haptoglobin in rheumatoid arthritis. J. Chromatogr. B 2011, 879, 1839-1843. [CrossRef] [PubMed]

23. Grimstein, C.; Choi, Y.K.; Wasserfall, C.H.; Satoh, M.; Atkinson, M.A.; Brantly, M.L.; Campbell-Thompson, M.; Song, S. $\alpha 1$ antitrypsin protein and gene therapies decrease autoimmunity and delay arthritis development in mouse model. J. Transl. Med. 2011, 9, 21. [CrossRef] [PubMed]

24. Elshikha, A.S.; Lu, Y.; Chen, M.J.; Akbar, M.; Zeumer, L.; Ritter, A.; Elghamry, H.; Mahdi, M.A.; Morel, L.; Song, S. $\alpha 1$ antitrypsin inhibits dendritic cell activation and attenuates nephritis in a mouse model of lupus. PLoS ONE 2016, 11, e0156583. [CrossRef] [PubMed]

25. Ciobanu, A.; Ciobanu, I.R.; Ionita, A.; Stoicescu, M.; Stroescu, I.; Caralicea, M.; Curut, O. Some humoral immunological aspects of the rheumatoid arthritis correlated with the ultrastructural changes of the rheumatoid synovial fluid. Romanian J. Morphol. Embryol. 1992, 38, 143-154.

26. Stefanescu, M.; Szegli, G.; Cremer, L.; Zarma, L.; Mazilu, E.; Naghiu, M.; Niculescu, D.; Gaches, A.; $\mathrm{Onu}, \mathrm{A}$. The presence and significance of some anti-enzyme antibodies (anti-plasminogen, anti-trypsin, anti-phospholipase C) in rheumatoid arthritis (RA) and reactive arthritis (RA). Arch. Roum. Pathol. Exp. Microbiol. 1989, 48, 47-53. [PubMed]

27. Struthers, G.R.; Lewin, I.V.; Stanworth, D.R. IgA- $\alpha 1$ antitrypsin complexes in ankylosing spondylitis. Ann. Rheum. Dis. 1989, 48, 30-34. [CrossRef] [PubMed] 
28. Lacki, J.K.; Schochat, T.; Leszczynski, P.; Mackiewicz, S.H.; Muller, W. IgA- $\alpha 1$-antitrypsin complex in systemic lupus erythematosus: Preliminary report. Lupus 1995, 4, 221-224. [CrossRef] [PubMed]

29. Lacki, J.K.; Schochat, T.; Porawaska, W.; Mackiewicz, S.H.; Muller, W. Does the serum level of IgA- $\alpha 1$-antitrypsin complex correlate with radiological progression in early rheumatoid arthritis? A 3-year follow-up study. Rheumatol. Int. 1995, 14, 227-230. [CrossRef] [PubMed]

30. Silosi, I.; Silosi, C.A.; Boldeanu, M.V.; Cojocaru, M.; Biciusca, V.; Avramescu, C.S.; Cojocaru, I.M.; Bogdan, M.; FolcuTi, R.M. The role of autoantibodies in health and disease. Romanian J. Morphol. Embryol. 2016, 57, 633-638.

31. Binder, C.J.; Papac-Milicevic, N.; Witztum, J.L. Innate sensing of oxidation-specific epitopes in health and disease. Nat. Rev. Immunol. 2016, 16, 485-497. [CrossRef] [PubMed]

32. Schwartz-Albiez, R.; Monteiro, R.C.; Rodriguez, M.; Binder, C.J.; Shoenfeld, Y. Natural antibodies, intravenous immunoglobulin and their role in autoimmunity, cancer and inflammation. Clin. Exp. Immunol. 2009, 158 (Suppl. 1), 43-50. [CrossRef] [PubMed]

33. Sutti, S.; Jindal, A.; Locatelli, I.; Vacchiano, M.; Gigliotti, L.; Bozzola, C.; Albano, E. Adaptive immune responses triggered by oxidative stress contribute to hepatic inflammation in nash. Hepatology 2014, 59, 886-897. [CrossRef] [PubMed]

34. Aletaha, D.; Neogi, T.; Silman, A.J.; Funovits, J.; Felson, D.T.; Bingham, C.O., III; Birnbaum, N.S.; Burmester, G.R.; Bykerk, V.P.; Cohen, M.D.; et al. 2010 rheumatoid arthritis classification criteria: An american college of rheumatology/european league against rheumatism collaborative initiative. Arthritis Rheum. 2010, 62, 2569-2581. [CrossRef] [PubMed]

35. Arnett, F.C.; Edworthy, S.M.; Bloch, D.A.; McShane, D.J.; Fries, J.F.; Cooper, N.S.; Healey, L.A.; Kaplan, S.R.; Liang, M.H.; Luthra, H.S.; et al. The american rheumatism association 1987 revised criteria for the classification of rheumatoid arthritis. Arthritis Rheum. 1988, 31, 315-324. [CrossRef] [PubMed]

36. Vitali, C.; Bombardieri, S.; Jonsson, R.; Moutsopoulos, H.M.; Alexander, E.L.; Carsons, S.E.; Daniels, T.E.; Fox, P.C.; Fox, R.I.; Kassan, S.S.; et al. Classification criteria for Sjögren's syndrome: A revised version of the European criteria proposed by the American-European consensus group. Ann. Rheum. Dis. 2002, 61, 554-558. [CrossRef] [PubMed]

37. Hochberg, M.C. Updating the american college of rheumatology revised criteria for the classification of systemic lupus erythematosus. Arthritis Rheum. 1997, 40, 1725. [CrossRef] [PubMed]

38. Uen, Y.H.; Lin, K.Y.; Sun, D.P.; Liao, C.C.; Hsieh, M.S.; Huang, Y.K.; Chen, Y.W.; Huang, P.H.; Chen, W.J.; Tai, C.C.; et al. Comparative proteomics, network analysis and post-translational modification identification reveal differential profiles of plasma con a-bound glycoprotein biomarkers in gastric cancer. J. Proteom. 2013, 83, 197-213. [CrossRef] [PubMed]

39. Huo, X.; Wu, B.; Feng, M.; Han, B.; Fang, Y.; Hao, Y.; Meng, L.; Wubie, A.J.; Fan, P.; Hu, H.; et al. Proteomic analysis reveals the molecular underpinnings of mandibular gland development and lipid metabolism in two lines of honeybees (Apis mellifera ligustica). J. Proteome Res. 2016, 15, 3342-3357. [CrossRef] [PubMed]

40. Liao, C.C.; Chou, P.L.; Cheng, C.W.; Chang, Y.S.; Chi, W.M.; Tsai, K.L.; Chen, W.J.; Kung, T.S.; Tai, C.C.; Lee, K.W.; et al. Comparative analysis of novel autoantibody isotypes against citrullinated-inter- $\alpha$-trypsin inhibitor heavy chain 3 (ITIH3) ${ }^{542-556}$ peptide in serum from taiwanese females with rheumatoid arthritis, primary Sjögren's syndrome and secondary Sjögren's syndrome in rheumatoid arthritis. J. Proteom. 2016, 141, 1-11.

41. Weber, D.; Milkovic, L.; Bennett, S.J.; Griffiths, H.R.; Zarkovic, N.; Grune, T. Measurement of HNE-protein adducts in human plasma and serum by ELISA-comparison of two primary antibodies. Redox Biol. 2013, 1, 226-233. [CrossRef] [PubMed]

(C) 2017 by the authors. Licensee MDPI, Basel, Switzerland. This article is an open access article distributed under the terms and conditions of the Creative Commons Attribution (CC BY) license (http:/ / creativecommons.org/licenses/by/4.0/). 